\title{
Treatment of Advanced Glaucoma Through Integrated Medicine
}

Wang $\mathrm{J}^{\star}$, Guo R, Su C and Shen W

Department of ophthalmology, Tianjin Medical University Eye Hospital \& Eye Institute, Tianjin, China

* Corresponding author: Jiantao Wang, Department of ophthalmology, Tianjin Medical University Eye Hospital \& Eye Institute, No. 251 Fukang Road, Nankai District, Tianjin 300384, China, Tel: 0086-022-58280810, 0086-022-23540682; E-mail: wangjiantao65@126.com

Received date: Dec 30, 2015; Accepted date: March 18, 2016; Published date: March 28, 2016

Copyright: (C) 2016 Wang J, et al. This is an open-access article distributed under the terms of the Creative Commons Attribution License, which permits unrestricted use, distribution, and reproduction in any medium, provided the original author and source are credited.

\section{Summary}

Glaucoma is a neurodegenerative disease characterized by the progressive loss of retinal ganglion cells and optic nerve axons. It is accompanied by visual field sensitivity loss and, eventually, irreversible visual loss [1]. Advanced glaucoma causes visual field concentric narrowing, which leads to the formation of a tubular visual field. The characteristics of advanced glaucoma are complicated, making its treatment difficult. Thus, a comprehensive evaluation of the preoperative risk of glaucoma is needed. This paper presents the use of integrated medicine to treat advanced glaucoma.

Keywords: Advanced glaucoma; Integrated medicine

\section{Case Report}

A 47-year-old woman was admitted to our department on an emergency basis because of a sudden onset of vision loss without eye pain, nausea, and vomiting. We diagnosed it as secondary glaucoma (Oculus laevus nearly absolute phase). The woman had a history of uveal effusion syndrome in both eyes, and she underwent cataract extraction, vitrectomy, and silicone oil filling in the right eye. The right eye became blind after the removal of silicone oil. Posterior scleral incision surgery was performed to prevent vision loss in the left eye. The surgery induced a high intraocular pressure, which was controlled by Mikelan. She had no family history of glaucoma, and she was under systemic hormone treatment for nearly 6 years because of kidney disease.

In the ophthalmic examination, the visual acuity was OD: hand move and left eye (OS): 20/50. The intraocular pressure was $17 \mathrm{mmHg}$ $\mathrm{OD}$ and $35 \mathrm{mmHg}$ OS that both eyes received a therapeutic regimen of drugs. Slit lamp biomicroscopy revealed iris segmental atrophy, posterior temporal synechiae, and mild opacity of the lens cortex in OS (Figure 1).

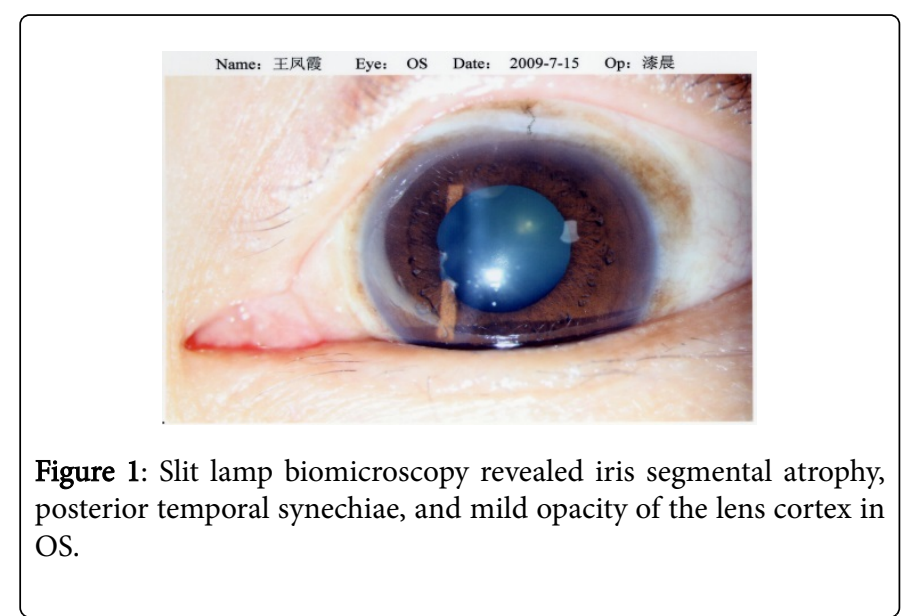

while clear cornea in both eyes (OU). Funduscopic examination presented a cup-disc ratio of 1.0 and a white disc in OS (Figure 2).

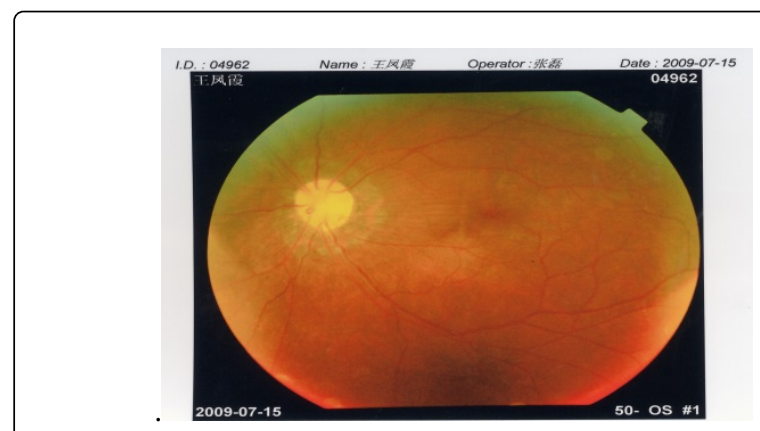

Figure 2: Funduscopic examination presented a cup-disc ratio of 1.0 and a white disc in OS.

Gonioscopy using the van Herick method and $360^{\circ}$ opened angle in OS showed that the anterior chambers were shallow (grade I). Ultrasound biomicroscopy presented an anterior chamber depth of $2.467 \mathrm{~mm}$ and a slight swelling of the iris (Figure 3). 
Citation: Wang J, Guo R, Su C, Shen W (2016) Treatment of Advanced Glaucoma Through Integrated Medicine. J Cytol Histol 7: 400. doi:

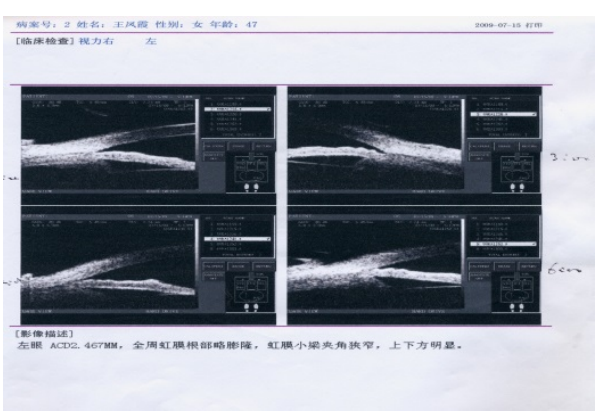

Figure 3: Ultrasound bio microscopy presented an anterior chamber depth of $2.467 \mathrm{~mm}$ and a slight swelling of the iris. 4).

Ocular ultrasonography verified that the retina is in position (Figure

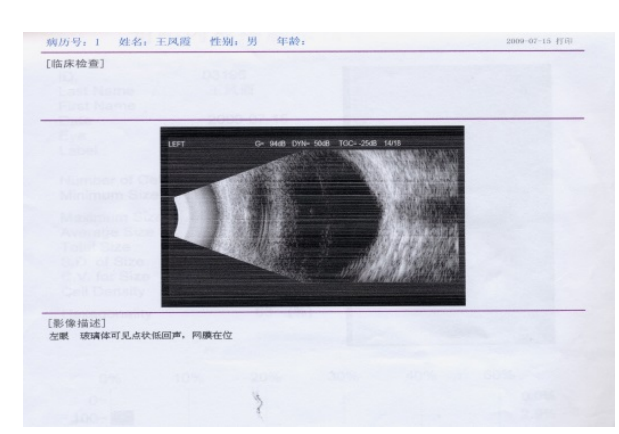

Figure 4: Ocular ultrasonography verified that the retina is in position.

We performed combined trabeculectomy, posterior scleral marsupialization, and vitreous aspiration, accompanied by local and systemic hormone therapy and hemostasis blood circulation treatment. Examination on the first postoperative day showed an intraocular pressure of $31 \mathrm{mmHg}$, which decreased to $19 \mathrm{mmHg}$ during incisioning of a large pale yellow liquid assembled under the conjunctiva. Clear cornea in OS and an ACD of 2.5 CT were also observed on the first postoperative day (Figure 5).

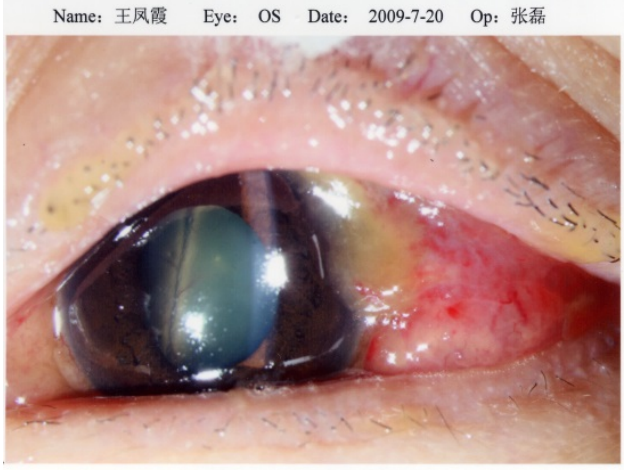

Figure 5: Clear cornea in OS and an ACD of $2.5 \mathrm{CT}$ were also observed on the first postoperative day.

Examination on the fifth postoperative day showed the expression of choroid, optic disc, and posterior of retina. Ocular ultrasonography displayed the detachment of the retina and choroid. We invited Professor Xiaoxin Li for a consultation and referral for further treatment (Figure 6).

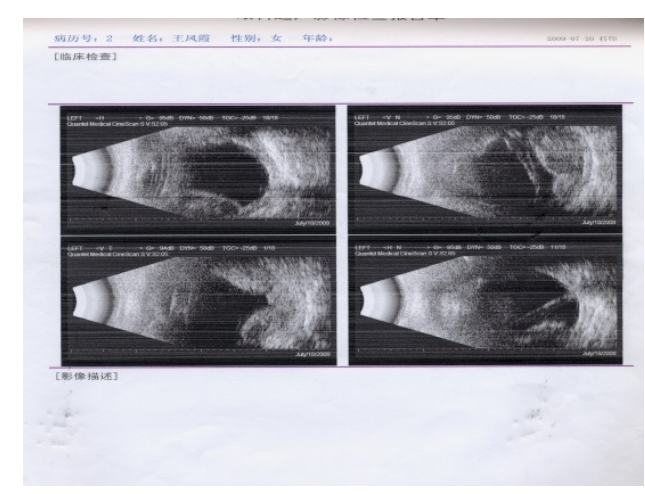

Figure 6: Ocular ultrasonography displayed the detachment of the retina and choroid.

\section{Discussion}

Many factors, such as age, family history of glaucoma, high myopia, diabetes, and hypotension, influence the defect tolerance ability of intraocular pressure in advanced glaucoma, in addition to high intraocular pressure or intraocular pressure fluctuation. Pure medication is insufficient to control a high intraocular pressure. Moreover, surgical treatment of patients with advanced glaucoma entails relatively large risks, such as surgical complications and limited visual function recovery. Thus, doctors often follow a cautious approach to surgical treatment. In this case, we carried out hospital consultation and clinical consultancy by using the idea of integrated medicine to evaluate the preoperative risk of glaucoma comprehensively. The purpose of this strategy is to find a safe and reliable method to treat patients with glaucoma, improve the quality of life of patients with advanced glaucoma, and reduce the pressure of society. As a general belief, a specialist is only a master of his own field and has insufficient knowledge of other major or all types of diseases. 
Citation: Wang J, Guo R, Su C, Shen W (2016) Treatment of Advanced Glaucoma Through Integrated Medicine. J Cytol Histol 7: 400. doi: 10.4172/2157-7099.1000400

Page 3 of 3

Integrated medicine has been utilized in other areas [2], particularly in the treatment of ophthalmologic diseases [3-5]. Therefore, the concept of integrated medicine in the treatment of glaucoma must be strengthened comprehensively to provide improved healthcare services to patients with glaucoma.

\section{Acknowledgments}

This article is supported by a grant from National Natural Science Foundation of China (81270994).

\section{References}

1. Kelliher C (2006) Trends in blind registration in the adult population of the republic of Ireland 1996-2003. Brit J Ophthalmol 90: 367-371.
2. Sharma A, Sharp DM, Walker IG, Manson JR (2008) Colorectal MDTs the teams perspective. Colorectal Dis 10: 63-68.

3. Zhe L, Guo-Li X, Yan-Ling W (2015) Establishment of holistic integrated medicine clinical thinking in ophthalmology physicians. Negative 6: 37-38.

4. Yan-Ling W (2013) Establishment of multidisciplinary diagnosis and treatment patterns for ocular ischemic syndrome. Chin J Ocul Fundus Dis 29.

5. Winterkorn JM, Beckman RI (1995) Receovery from ocular ischemic syndrome after treatment with verapamilj Neuroophthalmol 15: 209-211. 